\title{
Current Control System of the Power Supplies for LHD Superconducting Coils
}

\author{
Hirotaka Chikaraishi, Sigeyuki Takami, Tomoyuki Inoue, Satoru Sakakibara, Keisuke Matsuoka, Toshifumi Ise, \\ Daisuke Eto, Tomoyuki Haga, and the LHD experimental group
}

\begin{abstract}
The LHD is a fusion experimental facility using a large-scale superconducting coil system. The coil system includes six sets of superconducting coils, and six DC power supplies are used to charge them. For the current controllers of these power supplies, high accuracy of current control, fast response and robustness of the system are required. This paper describes the current control system for the LHD DC power supplies. First, the outline of the power system is presented, and then, the current controllers for the LHD are described. Finally, experimental results are presented and discussed in case of coil excitation using these control systems. The results show the various characteristics for each control system and indicate its possibility to control the system according to the requirements from a plasma experiment.
\end{abstract}

Index Terms-Converter control, DC power supplies, magnetic devices.

\section{INTRODUCTION}

$\mathbf{T}$ HE Large Helical Device (LHD) is a fusion experimental facility, which has a large-scale superconducting coil system, and it is now under operation at the National Institute for Fusion Science. This machine has twelve superconducting coils that form the magnetic field to confine the plasma. Regarding to the power supplies to excite these coils, six low voltage power supplies for steady state excitation were constructed and are now operating. For these power supplies, the following conditions were required; the steady state control error is less than $0.01 \%$ of the set value, the current settling time for $0.1 \%$ of control error is less than 1 second and the control system must be robust against turbulence caused by the plasma experiments. To resolve these requirements, some control systems are studied, designed and tested.

In the following sections, the outline of the control system for power supplies is introduced at first. Next, the estimation of circuit parameters needed to design a control system is described. Finally, the controller design and experimental results are presented.

\section{OUtLINE OF THE LHD POWER SySTEM}

Fig. 1 shows the connection of the coils and the power supplies. The twelve coils of LHD form three poloidal coil pairs and three helical coil pairs as shown in the figure, and they are

Manuscript received October 21, 2003.

H. Chikaraishi, S. Takami, T. Inoue, S. Sakakibara and K. Matsuoka are with the National Institute for Fusion Science, Oroshi 322-6, Toki, Gifu, Japan (e-mail: hchikara@nifs.ac.jp).

T. Ise and D. Eto are with Osaka University.

T. Haga is with the Shinko-MEX Co. Ltd.

Digital Object Identifier 10.1109/TASC.2004.830635

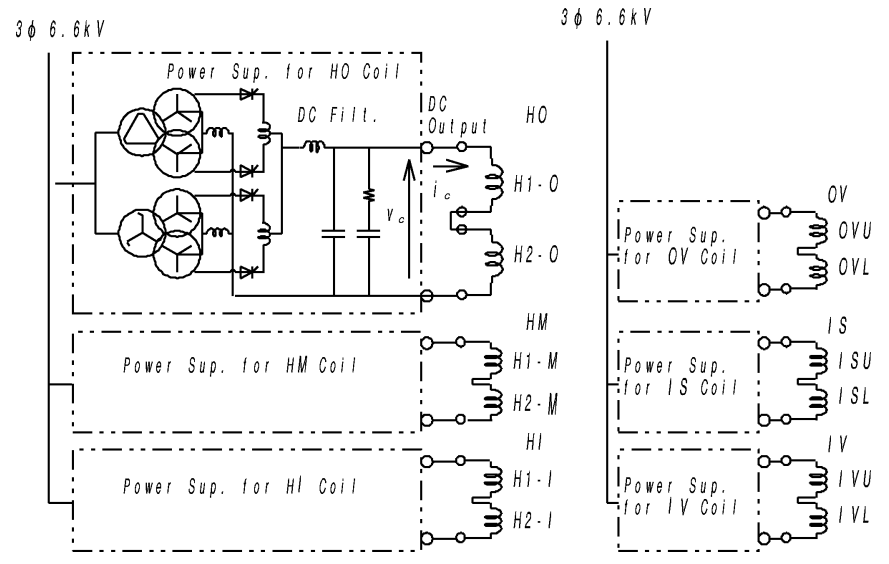

Fig. 1. Circuit diagram of power supplies and coils.

TABLE I

SPECIFICATIONS OF POWER SUPPLIES

\begin{tabular}{ccccc}
\hline \hline & HO, HM, HI & OV & IS & IV \\
\hline Voltage [V] & 45 & 33 & 33 & 33 \\
Current [kA] & 17.3 & 23.5 & 16.3 & 15.7 \\
\hline \hline
\end{tabular}

TABLE II

ACCURACIES OF CURRENT CONTROL

\begin{tabular}{cr}
\hline \hline Current measurement error & $<(0.03 \%$ of reading value $+4.5 \mathrm{~A})$ \\
Current control error & $<(0.04 \%$ of set value $+6 \mathrm{~A})$ \\
Current overshoot & not allowed \\
\hline
\end{tabular}

designated as OV, IS, IV, HO, HM and HI coils. Each power supply has a current sensor and a local voltage regulator, which controls the output voltage of power supplies. With this local regulator, the voltage drop in the circuit and a line voltage fluctuation are compensated, and each power supply can be handled as an ideal controlled voltage source. Table I shows the specifications of the power supplies.

A current control program, which controls all power supplies, is installed as a software in a computer system that connected to the power supplies with communication lines. To the current controller, specifications shown in Table II are required. This program keeps 4 different current controllers as subroutines and each controller is described with state vector as following;

$$
\begin{gathered}
s X=A X+\left[B_{1}, B_{2}, B_{3}\right]\left[\begin{array}{c}
I_{c}^{*} \\
I_{c} \\
s I_{p}
\end{array}\right], \\
V_{c}^{*}=C X+\left[D_{1}, D_{2}, D_{3}\right]\left[\begin{array}{c}
I_{c}^{*} \\
I_{c} \\
s I_{p}
\end{array}\right],
\end{gathered}
$$




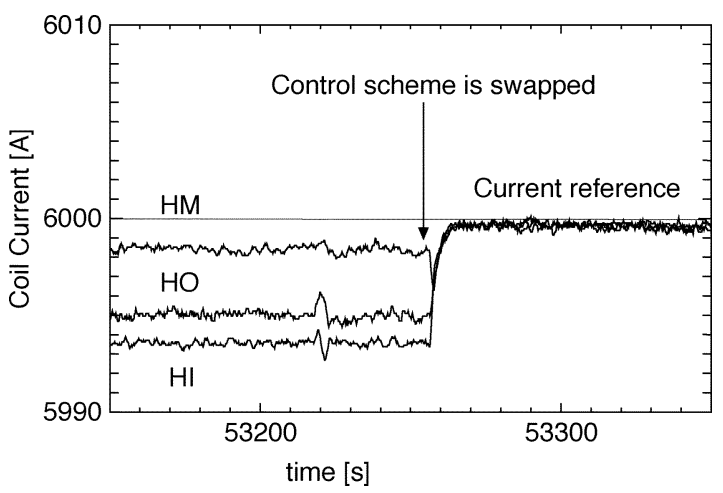

Fig. 2. Swap of control scheme from a moderate gain $P$ controller to a high gain $P$ controller.

where $X$ is a state vector, $A, B, C$ and $D$ are the matrix of the control gains. $I_{c}^{*}, I_{c}, I_{p}$ and $V_{c}^{*}$ demote the current reference, the actual coil current, the plasma current and the output voltage reference, respectively.

For some LHD plasma experiments such as a plasma current feedback operation, a current control scheme as used in steady state plasma operations cannot satisfy the requirements of dynamic characteristics and it is necessary to change the control scheme.

To swap a control scheme, internal variables in the controller must be initialized to suitable values to avoid the large voltage swing in transient state. Under the condition that $s X=0, s I_{p}=$ 0 and the current control error is small enough, the state variable $X$ becomes as following;

$$
X_{0}=[U-A]^{-1}\left[B_{1}+B_{2}\right] I_{c}^{*},
$$

where $U$ is a unit matrix. Therefore $X_{0}$ should be used as an initial value of $X$.

For the $P I$ control scheme, matrix $[U-A]$ becomes singular and $X_{0}$ cannot be derived with above equation. In this case, the state equations are transformed into the following forms, and the initial vector $X_{0}$ becomes calculable;

$$
\begin{aligned}
{\left[\begin{array}{c}
X \\
X_{i}
\end{array}\right] } & =\left[\begin{array}{cc}
A_{1} & A_{2} \\
A_{3} & U
\end{array}\right]\left[\begin{array}{c}
X \\
X_{i}
\end{array}\right]+\left[B_{1}, B_{2}\right]\left[\begin{array}{c}
I_{c}^{*} \\
I_{c}
\end{array}\right] \\
V & =\left[C_{1} C_{2}\right]\left[\begin{array}{c}
X \\
X_{i}
\end{array}\right]+\left[D_{1}, D_{2}\right]\left[\begin{array}{c}
I_{c}^{*} \\
I_{c}
\end{array}\right] \\
{\left[\begin{array}{c}
X_{0} \\
X_{i 0}
\end{array}\right] } & =\left[\begin{array}{c}
{\left[U-A_{1}\right]^{-1}\left[B_{1}+B_{2}\right] I_{c}^{*}} \\
0
\end{array}\right] .
\end{aligned}
$$

Fig. 2 shows an example of a waveform when the control scheme was swapped from a moderate gain $P$ control to a high gain $P$ control. Even though the moderate gain $P$ control causes a steady state error and it becomes an initial offset for high gain controller, each control scheme is designed as stable enough and does not cause any problem. With the ability to swap the current control scheme under the condition of the coil magnetization, the power system becomes more useful for plasma experiments.

\section{PARAMETER Estimation OF COIL System}

In the design of a control system, the electrical parameters of the superconducting coil system are required to determine the control gains mentioned above. The inductance matrix of superconducting coils was measured from the coil terminals [2].

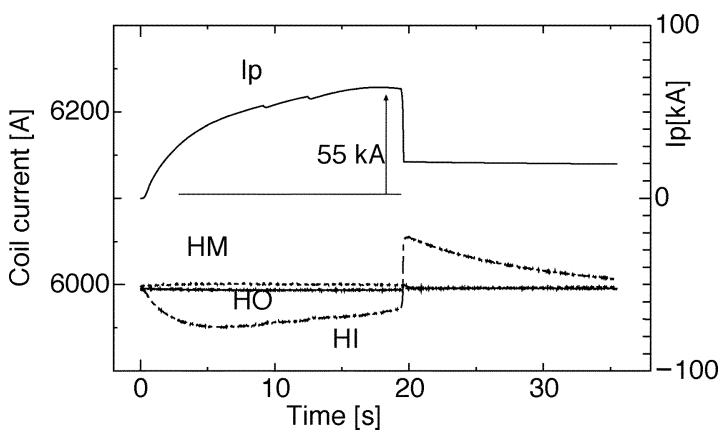

Fig. 3. Induction caused by plasma current, where $P$ controller with $k=0.1$ is used.

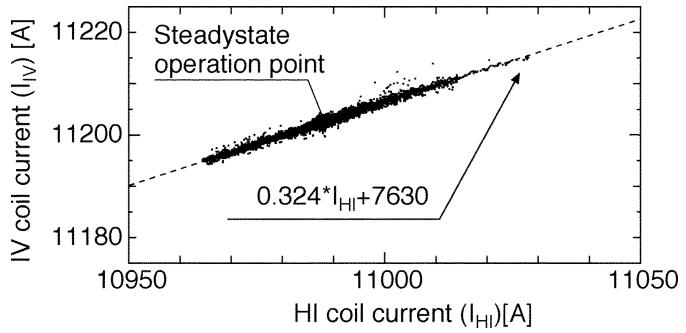

Fig. 4. Plots of the coil current deviation when plasma experiments with a plasma current were done.

Essentially helical type fusion plasma does not require plasma current but operations with plasma current are useful to study plasma dynamics. With this reason, some plasma experiments with plasma current were executed in the LHD.

Fig. 3 shows the induction caused by the plasma current when the $P$ controller with $k=0.1$, which will be mentioned in the following section, is used. In this figure, the curve of plasma current does not return to zero after a plasma operation because the signal of $d i_{p} / d t$ becomes too large and it exceeds the dynamic range of the system.

In this experiment, the induction to the coil current is observed only on the $\mathrm{HI}$ coil because the fast changing of the magnetic flux is shielded by the HI coil current. In this shot, the plasma current rises to $55 \mathrm{kA}$ in 10 seconds and shuts down suddenly. The induced voltage caused by the plasma current change decreases the HI coil current at about $70 \mathrm{~A}$ if no current control is applied. This current deviation was detected by the coil current control system and the coil terminal voltages were induced to suppress this deviation. With this current control, the current deviation of $\mathrm{HI}$ coil is suppressed with a time constant of $10 \mathrm{~s}$ and the magnetic flux pass the HI coil. For HM and HO coils, interlinked flux changes with a time constant of $10 \mathrm{~s}$ and the induced voltage caused by this flux can be cancelled by the current regulator. Therefore, these two coils did not show significant current changes. When the plasma current disrupts, its induction increases the $\mathrm{HI}$ coil current.

Fig. 4 shows plots of the coil current deviation when plasma experiments with a plasma current were done. The horizontal axis is for the $\mathrm{HI}$ coil and the vertical axis is for the IV coil, and the point of $I_{H I}=11.0 \mathrm{kA}$ and $I_{I V}=11.2 \mathrm{kA}$ is a steady state operation point. The plots exist surround a line with slope of 0.324 as shown in the figure. This means that the ratio of mutual coupling between plasma, HI and IV coils is constant, and the plasma can be described with a rigid coil when a control system 
TABLE III

Mutual Inductance Between CoIls and Plasma

\begin{tabular}{lcrrrr}
\hline \hline \multicolumn{6}{c}{ Mutual inductance [unit mH] } \\
\hline $\mathrm{HO}$ & $\mathrm{HM}$ & $\mathrm{HI}$ & $\mathrm{OV}$ & IS & IV \\
1.60 & 1.69 & 1.80 & 1.05 & 0.88 & 0.67 \\
\hline \hline
\end{tabular}

is designed. The mutual inductances between plasma and coils are estimated by the following way.

When the plasma current is cut off, the interlinked flux to the coils should be kept constant because the time constant of the coil current control is sufficiently longer than the plasma current decay time. Therefore, the circuit equation in this situation is described as follows;

$$
L_{c} i_{c}+M_{c p} i_{p}=\text { constant }
$$

where $L_{c}, M_{c p}, i_{c}$ and $i_{p}$ are self-inductance of coils, mutual inductance between coils and plasma, coil current and plasma current respectively. With this relation, the mutual inductance between plasma and coils were estimated. Table III shows the estimated mutual inductances and they are about $70 \%$ of the calculated values using a plasma model with a line current. These differences between the experimental value and the calculated value may be caused by the difference of current distribution in the actual plasma and it in the circuit model.

\section{Designed CurRent Control Schemes}

\section{A. PI Controller}

First, the $P I$ control scheme, which does not use a signal of the plasma current, is designed for this system. Although this control scheme is simple, it has an adequate performance for the steady state current control.

The current regulator used in this system is formed as follows;

$$
V_{c}^{*}=\frac{k}{s} R_{c}\left(I_{c}^{*}-I_{c}\right)+k L_{c}\left(I_{c}^{*}-I_{c}\right),
$$

where $L_{c}$ is an inductance matrix of the superconducting coils, $R_{c}$ is a resistance matrix, and $\mathrm{k}$ is the scalar control gain. From the equations, the control matrix in (1) and (2) are evaluated as follows;

$$
\begin{aligned}
& A_{c}=0, \quad B_{c}=\left[\begin{array}{cc}
U & 0 \\
0 & -U
\end{array}\right], \\
& C_{c}=k R_{c}, \quad D_{c}=k\left[\begin{array}{cc}
L_{c} & 0 \\
0 & -L_{c}
\end{array}\right] .
\end{aligned}
$$

The step response of the coil current is as following;

$$
I_{c}=I_{c 0}+\frac{1}{1+s \tau}\left(I_{c}^{*}-I_{c 0}\right),
$$

where $\tau=1 / k$ is a characteristic time constant, $I_{c 0}$ is an initial value of $I_{c}$. From this equation, it is clear that every coil current has the same response time and the balance of the coil currents is kept constant during the transient.

The control gain $k$ determining system response has an upper limit for the stability. This limit is determined due to the control theory as $k<2 \pi f_{c} / 4$, where $f_{c}$ is a cut-off frequency of a low pass filter inserted to reduce noise. In the LHD power system, the $f_{c}$ is selected as $0.87 \mathrm{~Hz}$, therefore the upper limit of $k$ is 1.3 .

The $P$ control scheme is obtained by the setting of $R_{c}=0$ in (7). In this $P$ control, the steady state control error $I_{e}=I_{c}^{*}-I_{c}$ remains as following;

\begin{tabular}{|c|c|c|c|c|}
\hline Control scheme & $\begin{array}{c}\mathrm{P} \\
k=0.1\end{array}$ & $\begin{array}{c}\text { PI } \\
k=1\end{array}$ & $\mathrm{H} \infty(1)$ & $\overline{\mathrm{H} \infty(2)}$ \\
\hline Size of $X$ & 6 & 12 & 68 & 68 \\
\hline $\begin{array}{l}\text { Steady state } \\
\text { error }\end{array}$ & $0.1 \%$ & $<0.01 \%$ & $<0.01 \%$ & $<0.01 \%$ \\
\hline $\begin{array}{c}\text { Response } \\
\text { time constant }\end{array}$ & $10 \mathrm{~s}$ & $1 \mathrm{~s}$ & $<0.3 \mathrm{~s}$ & $<0.3 \mathrm{~s}$ \\
\hline
\end{tabular}

$$
I_{e}=\left(k L_{c}\right)^{-1} \cdot R_{c} \cdot I_{c}^{*} \text {. }
$$

TABLE IV

COMPARISON OF THE CURRENT CONTROL SYSTEM

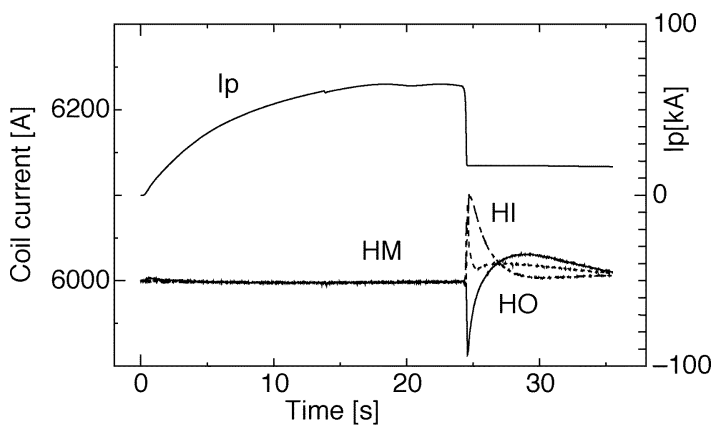

(a)

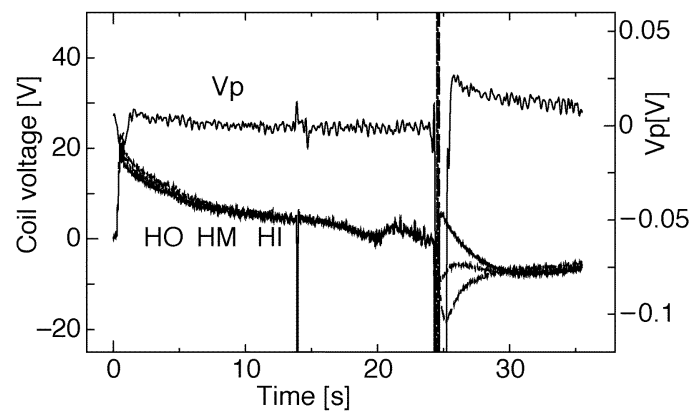

(b)

Fig. 5. Induction caused by plasma current. (a) Plasma and coil currents, when $H \infty$ (1) control is applied. (b) Induced voltage in plasma and coil terminal voltage, when $H \propto(1)$ control is applied.

In this system, $I_{e}$ may become about $0.01 \%$ of $I_{c}^{*}$ when $k=1.0$ because the orders of $R_{c}$ and $L_{c}$ are $10^{-4}$ and 1, respectively.

Table IV shows the size of state vector $X$ and some characteristic parameters for each control system. The $H \propto(1)$ and $H \infty(2)$ controllers will be described later. When we use the $P$ control, $k=1$ is necessary to satisfy the required accuracy and response time as shown in the Table IV.

\section{B. $H \infty$ Controller}

In the $P I$ controller mentioned above, the control gain is limited by the stability requirement and the response time constant cannot be small. The $H \infty$ design scheme is one of the solutions to manage both the robustness and fast response. We designed two of $H \infty$ controllers, which have different characteristics, for the LHD power system. One, named as $H \propto(1)$, is designed to keep the coil currents in constant even if the plasma current is excited. The another, named as $H \infty(2)$, is designed to keep magnetic flux constant during a plasma experiment.

Fig. 5 shows the induction caused by a plasma current when $H \infty$ (1) controller is applied and Fig. 6 shows the case when $H \infty(2)$ control is applied. In these figure, $V_{p}$ is the induced voltage to the plasma caused by the coil current change. Fig. 5(a) 


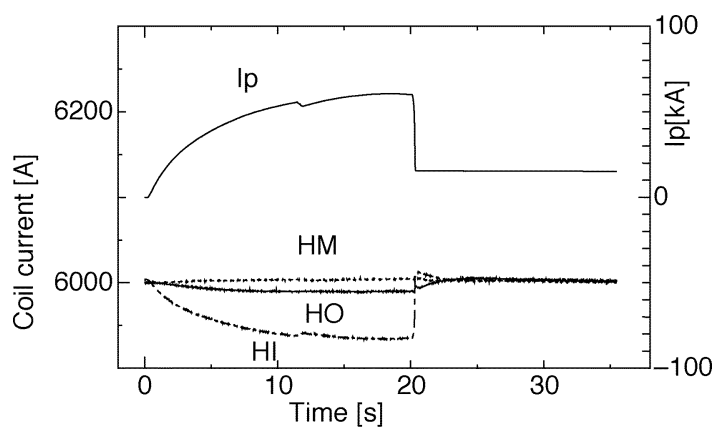

(a)

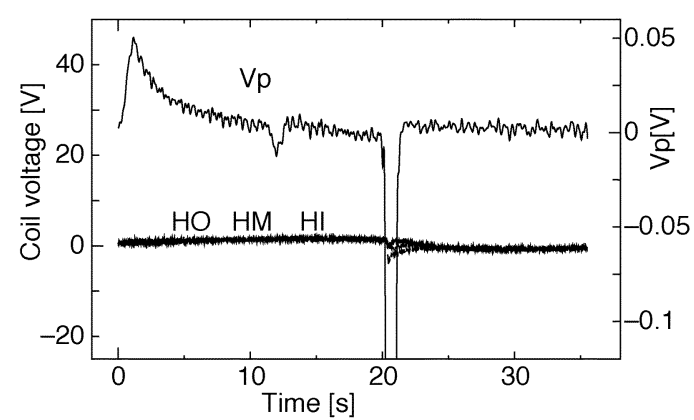

(b)

Fig. 6. Induction caused by plasma current. (a) Plasma and coil currents, when $H \propto$ (2) control is applied. (b) Induced voltage in plasma and coil terminal voltage, when $H \propto(2)$ control is applied.

shows that the coil currents were kept in constant while the plasma current ramps up and reaches flattop. When the plasma disrupts, the $d I p / d t$ signal exceeds the dynamic range of the power system, the coil currents show some transient waveforms but it does not cause any instability in the current control system. When $H \propto(1)$ control is used, the magnetic flux generated by the plasma current passes through the superconducting coils, therefore only a small one turn voltage is induced to the plasma as shown in Fig. 5(b). In Fig. 6, which uses $H \propto(2)$ control, the coil currents were changed to keep the flux constant and terminal voltages were kept at almost zero, and the induced voltage $V_{p}$ to the plasma accelerates the plasma current. When this scheme is applied to the LHD, it is clear that the rebound of the coil current during plasma shut down is smaller than in the case of Fig. 6 or Fig. 3, and the coil currents return to their references immediately.

\section{SUMMARY}

This paper describes the current control system in the LHD DC power system. To design the current controller, the electrical parameters of the coil system are estimated by experimental results and some types of controllers are designed. The test results show that these controllers are stable and they satisfy the requirements. The reaction caused by the plasma current is different for each control system, and the effect of difference in current response to the plasma characteristics will be studied.

\section{ACKNOWLEDGMENT}

The power system of the LHD superconducting coil system is constricted under the cooperation with Aichi electric co., ltd., Kobe steel co., ltd., Densan co., ltd. and Mitsubishi space software. In addition, LHD experiments are supported by many staffs of the project team. Moreover, this work is performed under the auspices of the NIFS Collaborative Research Program. The authors would like to thank the members of the LHD project and cooperating companies.

\section{REFERENCES}

[1] A. Iiyoshi et al., "Overview of the Large Helical Device project," $\mathrm{Nu}$ clear Fusion, vol. 39, pp. 1245-1256, 1999.

[2] H. Chikaraishi et al., "Estimation of electrical parameters of large scale superconducting coil system for fusion plasma experimental facility," IEEE Trans. Appl. Supercond., vol. 12, no. 1, pp. 1374-1377, March 2002.

[3] T. Ise et al., "Current control of magnetically coupled superconducting coils for nuclear fusion experimental system using $\mathrm{H}$ infinity control scheme," in Proc. 27th Annual IEEE Power Electronics Specialists Conf., 1996, pp. 1099-1104. 